\title{
Comprehensive investigation of clinicopathologic features, oncogenic driver mutations and immunohistochemical markers in peripheral lung squamous cell carcinoma
}

\author{
Yang Zhang ${ }^{1,2 *}$, Difan Zheng ${ }^{1,2 *}$, Yuan $\mathrm{Li}^{2,3}$, Yunjian $\operatorname{Pan}^{1,2}$, Yihua Sun ${ }^{1,2}$, Haiquan Chen ${ }^{1,2,4}$ \\ ${ }^{1}$ Department of Thoracic Surgery, Fudan University Shanghai Cancer Center, Shanghai, 200032, China; ${ }^{2}$ Department of Oncology, Shanghai \\ Medical College, Fudan University, Shanghai, 200032, China; ${ }^{3}$ Department of Pathology, Fudan University Shanghai Cancer Center, Shanghai, \\ 200032, China; Institutes of Biomedical Sciences, Fudan University, Shanghai 200032, China \\ Contributions: (I) Conception and design: Y Zhang, D Zheng, Y Sun, H Chen; (II) Administrative support: H Chen; (III) Provision of study materials \\ or patients: Y Sun, H Chen; (IV) Collection and assembly of data: Y Zhang, D Zheng, Y Li, Y Pan; (V) Data analysis and interpretation: Y Zhang, D \\ Zheng; (VI) Manuscript writing: All authors; (VII) Final approval of manuscript: All authors. \\ *These authors contributed equally to this work. \\ Correspondence to: Haiquan Chen, MD. Department of Thoracic Surgery, Fudan University Shanghai Cancer Center, 270 Dong-An Road, Shanghai \\ 200032, China. Email: hqchen1@yahoo.com; Yihua Sun. Department of Thoracic Surgery, Fudan University Shanghai Cancer Center, 270 Dong-An \\ Road, Shanghai 200032, China. Email: Sun_yihua76@hotmail.com.
}

Background: Although the majority of lung squamous cell carcinomas (SQCC) arise in central airways, the prevalence of peripheral (p) SQCC is increasing. This study aimed to have a comprehensive investigation of clinicopathologic features, status of common driver mutations and immunophenotypes of p-SQCC compared to central (c) SQCC.

Methods: A total of 261 p-SQCC were compared to 444 c-SQCC for clinicopathologic characteristics. Comprehensive mutational analysis of EGFR, KRAS, HER2, BRAF, PIK3CA, DDR2, AKT1, ALK, ROS1, RET and FGFRs were performed. TTF1, CK7, Napsin A and PE10 protein expression were analyzed through immunohistochemistry (IHC). TTF1, CK7, CK8, SPA and TP63 gene expression levels were measured by quantitative real-time PCR.

Results: Compared to c-SQCC, p-SQCC were associated with female $(14.2 \%$ vs. $4.5 \%, \mathrm{P}<0.001)$, never-smokers $(22.6 \%$ vs. $13.3 \%, \mathrm{P}=0.001)$, older age at diagnosis (64.9 vs. 59.5 years, $\mathrm{P}<0.001)$ and lower pathologic stage $(\mathrm{P}<0.001)$. The frequency of EGFR mutations was significantly higher in $\mathrm{p}-\mathrm{SQCC}$ than c-SQCC (6.2\% vs. 2.2\%, $\mathrm{P}=0.040)$. Positive protein expression of TTF1 ( $\mathrm{P}=0.010)$ and $\mathrm{CK} 7(\mathrm{P}=0.001)$ was significantly more prevalent in p-SQCC. p-SQCC had significantly higher gene expression of SPA $(\mathrm{P}=0.003)$, whereas c-SQCC showed higher gene expression of TP63 ( $\mathrm{P}=0.028)$.

Conclusions: Lung p-SQCC had distinctive clinicopathologic characteristics and molecular features compared to c-SQCC, but showed some similarity with adenocarcinoma (ADC).

Keywords: Peripheral lung squamous cell carcinoma (p-SQCC); clinicopathologic characteristics; driver mutations; immunohistochemical markers; gene expression

Submitted Jun 03, 2017. Accepted for publication Jul 27, 2017.

doi: $10.21037 /$ jtd.2017.10.43

View this article at: http://dx.doi.org/10.21037/jtd.2017.10.43

(C) Journal of Thoracic Disease. All rights reserved. 


\section{Introduction}

Squamous cell carcinoma (SQCC) along with adenocarcinoma (ADC), adenosquamous cell carcinoma and large cell carcinoma are the main histologic subtypes of nonsmall cell lung cancer. SQCCs have a close relation with cigarette smoking and are more likely to be centrally located. However, the prevalence of peripheral lung squamous cell carcinoma (p-SQCC) is increasing $(1,2)$. Previous studies have reported several distinctive features of p-SQCC (1-5). p-SQCC 2.0 centimeters $(\mathrm{cm})$ or less in diameter were reported to have very low incidence of lymph node involvement $(1,4,5)$. Sakurai and colleagues (3) investigated $70 \mathrm{p}$-SQCC of $3.0 \mathrm{~cm}$ or less in diameter, and found that none of the patients with $\mathrm{N} 2$ disease had skipping metastasis. However, survival did not differ significantly between P-SQCC and central (c) SQCC (2).

Our previous studies provided in vivo evidence of the transdifferentiation of lung ADC to SQCC. We showed mouse lung ADC could progressively transdifferentiate into SQCC $(6,7)$. As ADCs tend to be located in the peripheral lung, we hypothesized that p-SQCC might had some similarity with ADC compared to c-SQCC.

In this study, we carried out a comprehensive investigation of clinicopathologic features, prognosis, status of common driver mutations, as well as gene expression level and protein staining of "adenocarcinoma markers" and "squamous marker" of p-SQCC in comparison to c-SQCC. We also assessed the morphological characteristics of p-SQCC and their associations with survival.

\section{Methods}

From October 2007 to November 2014, we reviewed a series of lung squamous cell carcinoma patients who underwent surgical resection at the Department of Thoracic Surgery, Fudan University Shanghai Cancer Hospital, Shanghai, China. Our hospital is a university teaching hospital as well as a tertiary care referral hospital. Squamous cell carcinoma with $<10 \%$ glandular component was still classified as "squamous cell carcinoma" since adenosquamous carcinoma is classified as carcinoma containing at least $10 \%$ each of malignant squamous and glandular components according to the World Health Organization classification (8). Tumors with more than $10 \%$ TTF1 and/or CK7 positive tumor cells were not included in this study. Peripheral squamous cell carcinoma was defined as tumor located in or more peripheral to the subsegmental bronchus. We prospectively collected the following clinicopathologic characteristics: gender, age, history of smoking, tumor location, type of surgical resection, tumor size, tumor-nodemetastasis (TNM) stage according to the seventh edition of lung cancer staging system (9), postoperative adjuvant chemotherapy and radiotherapy which were recommended in line with the National Comprehensive Cancer Network (NCCN) guidelines, recurrence-free survival (RFS) and overall survival (OS). The survival information was obtained through follow up clinic or telephone.

Pathologic slides of 119 p-SQCC samples were reviewed for morphologic features. According to the 2015 World Health Organization Classification, we classified p-SQCC into keratinizing, nonkeratinizing and basaloid subtypes (10). Based upon the growth pattern, p-SQCCs were classified as the alveolar space-filling type, the expanding type and the combined type (2). The definitions of "tumor budding" and "single-cell invasion" were previously described (11).

This study was conducted in line with the Helsinki Declaration. The Institutional Review Board of Fudan University Shanghai Cancer Center approved this study (IRB\#090977-1). All patients provided written informed consent.

\section{Mutational analysis}

Briefly, after frozen tumor specimens were dissected into TRIzol (Invitrogen), RNA was extracted as per standard protocol, and was reverse transcribed into cDNA. EGFR (exons 18-22), KRAS (exons 2-3), HER2 (exons 18-21), $B R A F$ (exons 11-15), PIK3CA (exon 9 and exon 20), AKT1 (exons 2-3) and DDR2 (whole coding exons) were amplified by PCR using cDNA. The amplified products were then analyzed by direct sequencing. For the detection of $A L K$, ROS1, RET and FGFR (including FGFR1, FGFR 2 and $F G F R 3$ ) fusions, we designed multiple pairs of primers to cover all the known fusion variants (12-14).

\section{TTF1, CK7, Napsin A and PE10 immunobistochemistry (IHC)}

Protein expression of TTF1, CK7, Napsin A and PE10 were assessed by IHC. Briefly, paraffin-embedded tissue sections were first deparaffinized and rehydrated. After antigen retrieval, sections were incubated with primary anti-TTF1 antibody (8G7G3/1, DAKO, 1:100), antiCK7 antibody (OV-JL 12/30, MXB, 1:100), anti-Napsin A antibody (KCG1.1, Abcam, 1:500), and anti-PE10 antibody 
Table 1 Clinicopathologic characteristics of p-SQCC compared to c-SQCC

\begin{tabular}{|c|c|c|c|}
\hline Variables & $\begin{array}{c}\text { p-SQCC } \\
(n=261)\end{array}$ & $\begin{array}{c}\text { c-SQCC } \\
(n=444)\end{array}$ & $P$ \\
\hline Gender & & & $<0.001$ \\
\hline Female & $37(14.2 \%)$ & $20(4.5 \%)$ & \\
\hline Male & $224(85.8 \%)$ & $424(95.5 \%)$ & \\
\hline Age (years) & & & $<0.001$ \\
\hline Mean & 64.9 & 59.5 & \\
\hline SD & 9.0 & 7.8 & \\
\hline Smoking history & & & 0.001 \\
\hline Never & $59(22.6 \%)$ & $59(13.3 \%)$ & \\
\hline Ever & $202(77.4 \%)$ & $385(86.7 \%)$ & \\
\hline Surgical resection & & & $<0.001$ \\
\hline Wedge/segmentectomy & $6(2.3 \%)$ & $0(0)$ & \\
\hline Lobectomy & $244(93.5 \%)$ & $165(37.2 \%)$ & \\
\hline Bi-lobectomy & $10(3.8 \%)$ & $78(17.6 \%)$ & \\
\hline Sleeve lobectomy & $0(0)$ & $83(18.7 \%)$ & \\
\hline Pneumonectomy & $1(0.4 \%)$ & $118(26.6 \%)$ & \\
\hline Tumor size (cm) & & & 0.494 \\
\hline Mean & 4.3 & 4.3 & \\
\hline SD & 2.0 & 2.2 & \\
\hline Pathologic stage & & & $<0.001$ \\
\hline 1 & $144(55.2 \%)$ & $156(35.1 \%)$ & \\
\hline ॥ & $53(20.3 \%)$ & $144(32.4 \%)$ & \\
\hline III & $64(24.5 \%)$ & $144(32.4 \%)$ & \\
\hline
\end{tabular}

p-SQCC, peripheral lung squamous cell carcinoma; c-SQCC, central lung squamous cell carcinoma; SD, standard deviation.

(PE10, Changdao, 1:50). Protein staining was scored as "positive" when there was any staining.

\section{TTF1, CK7, CK8, SPA and TP63 gene expression levels}

TTF1, CK7, CK8, SPA and TP63 gene expression levels were measured by quantitative real-time PCR (qRT-PCR) using cDNA extracted from p-SQCC, c-SQCC as well as normal lung samples. The expression level of the housekeeping gene $\beta$-actin was calculated as an internal reference. Normalized expression value was calculated using the formula: $2^{[\mathrm{CT} \text { (Normal) - CT ( } \beta \text {-actin)] - [CT (Tumor) - CT ( } \beta \text {-actin)] }}$. Median expression level of all tumor samples was used as the cutoff value for separating high and low expression.

\section{Statistical analysis}

Correlations between two categorical variables were assessed using Pearson's chi-squared test or Fisher's exact test. We applied independent sample $t$-test to determine the association between a categorical variable and a continuous variable. Kaplan-Meier method with log-rank tests were used for comparisons of RFS and OS in univariate analysis. Multivariate survival analysis was performed using Cox proportional hazards regression. The statistical analysis was conducted in SPSS 16.0 (SPSS Inc., Chicago, IL, USA). All tests were two tailed, and $\mathrm{P}<0.05$ was considered as statistically significant.

\section{Results}

\section{Clinical characteristics}

There were a total of 261 p-SQCCs out of 705 SQCCs, accounting for a proportion of $37.0 \%$ (Table 1). Compared to c-SQCC, p-SQCC had a significantly higher percentage of female $(14.2 \%$ vs. $4.5 \%, \mathrm{P}<0.001)$ and never-smokers (22.6\% vs. $13.3 \%, \mathrm{P}=0.001$ ). Patients with $\mathrm{p}-\mathrm{SQCC}$ were significantly older than those with c-SQCC (mean age: 64.9 vs. 59.5 years, $\mathrm{P}<0.001$ ). Patients with c-SQCC tended to receive more extensive surgeries. Patients with p-SQCC were associated with earlier stage at diagnosis than those with c-SQCC $(\mathrm{P}<0.001)$. There was no significant difference regarding RFS or OS between p-SQCC and c-SQCC (Figure 1). The median 3-year RFS rate for p-SQCC and c-SQCC was $58.2 \%$ and $55.4 \%$, respectively. The median 3-year OS rate for p-SQCC and c-SQCC was $71.0 \%$ and $70.1 \%$, respectively. We further compared survival in stage I, II or III patients separately, RFS and OS were not statistically significant in each stage category. In multivariate Cox regression model adjusted for gender, age, smoking history, type of surgical resection, pathologic stage and postoperative adjuvant chemotherapy and radiotherapy, peripheral tumor location was not a significant predictor for RFS or OS.

\section{Status of common driver mutations}

We performed comprehensive mutational analysis in a series of 413 lung SQCCs including 145 p-SQCCs and 268 c-SQCCs (Figure 2 and Table 2). In patients with p-SQCC, 

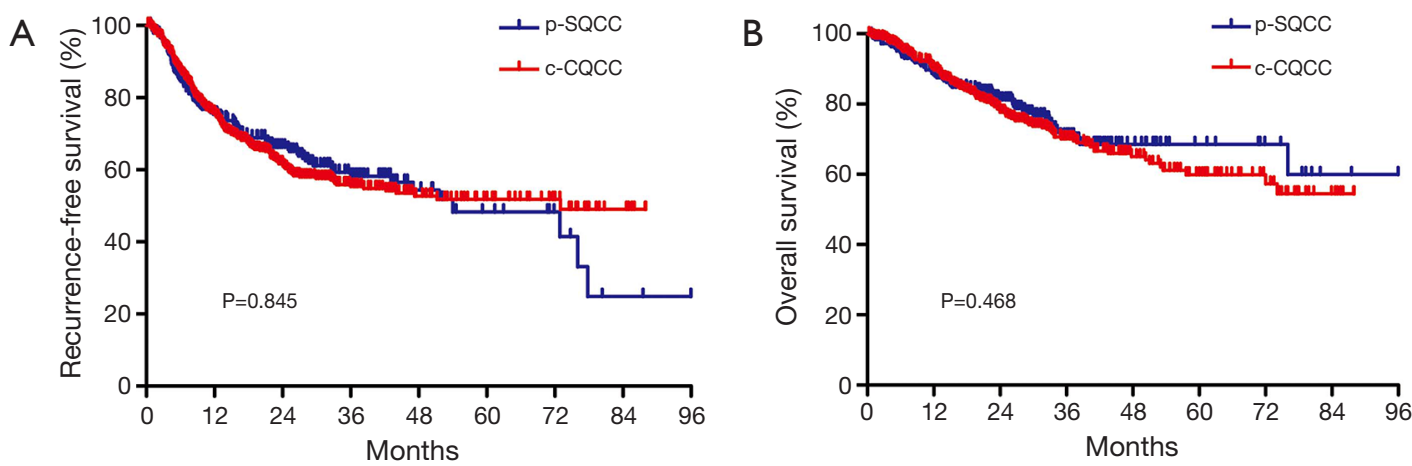

Figure 1 Comparison of recurrence-free survival (A) and overall survival (B) between peripheral lung squamous cell carcinoma (p-SQCC) and central lung squamous cell carcinoma (c-SQCC).
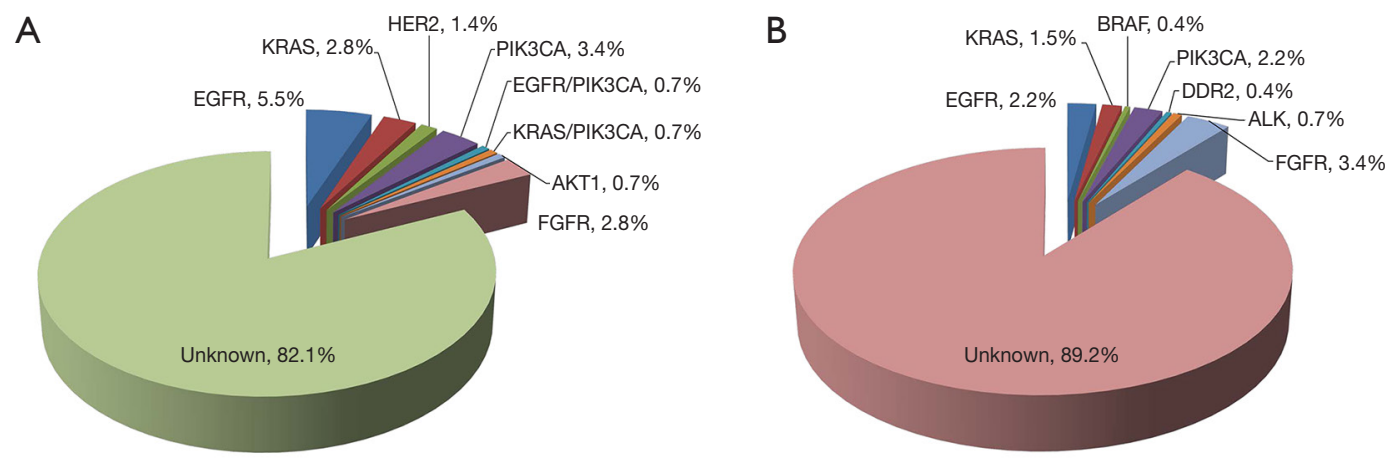

Figure 2 Spectrum of common driver mutations in peripheral lung squamous cell carcinoma (A) and central lung squamous cell carcinoma (B).

Table 2 Frequency of driver mutations in p-SQCC compared to c-SQCC

\begin{tabular}{lccc}
\hline Driver mutations & $\mathrm{p}$-SQCC $(\mathrm{n}=145)$ & $\mathrm{c}-\mathrm{SQCC}(\mathrm{n}=268)$ & $\mathrm{P}$ \\
\hline EGFR & $9(6.2 \%)$ & $6(2.2 \%)$ & 0.040 \\
KRAS & $5(3.4 \%)$ & $4(1.5 \%)$ & 0.288 \\
HER2 & $2(1.4 \%)$ & $0(0)$ & 0.123 \\
BRAF & $0(0)$ & $1(0.4 \%)$ & 1.000 \\
PIK3CA* & $7(4.8 \%)$ & $6(2.2 \%)$ & 0.150 \\
DDR2 & $0(0)$ & $1(0.4 \%)$ & 1.000 \\
AKT1 & $1(0.7 \%)$ & $0(0)$ & 0.351 \\
ALK & $0(0)$ & $2(0.7 \%)$ & 0.543 \\
FGFR & $4(2.8 \%)$ & $9(3.4 \%)$ & 1.000 \\
All & $26(17.9 \%)$ & $29(10.8 \%)$ & 0.042 \\
\hline * one PIK3CA mutation was concurrent with EGFR mutation
\end{tabular}

*, one PIK3CA mutation was concurrent with EGFR mutation, one PIK3CA mutation was concurrent with KRAS mutation. p-SQCC, peripheral lung squamous cell carcinoma; C-SQCC, central lung squamous cell carcinoma. there were 9 (6.2\%) EGFR mutations, 5 (3.4\%) KRAS mutations, 2 (1.4\%) HER2 mutations, 7 (4.8\%) PIK3CA mutations (1 concurrent with EGFR mutation, 1 concurrent with KRAS mutation), 1 (0.7\%) AKT1 mutation and $4(2.8 \%) F G F R$ fusions. In patients with c-SQCC, there were 6 (2.2\%) EGFR mutations, 4 (1.5\%) KRAS mutations, 1 (0.4\%) BRAF mutation, 6 (2.2\%) PIK3CA mutations, $1(0.4 \%)$ DDR2 mutation, 2 (0.7\%) ALK fusions and $9(3.4 \%)$ FGFR fusions. The frequency of EGFR mutations was significantly higher in $\mathrm{p}$-SQCC than c-SQCC $(\mathrm{P}=0.040)$. The proportion of patients with a known oncogenic driver mutation was also significantly higher in p-SQCC than c-SQCC (17.9\% vs. 10.8\%, $\mathrm{P}=0.042)$.

\section{Expression of "adenocarcinoma markers" and "squamous marker"}

We first performed IHC analysis of "adenocarcinoma markers" including TTF1, CK7, Napsin A and PE10 in p-SQCC and c-SQCC (Table 3). A significantly higher 
Table 3 Positive immunohistochemical staining of "adenocarcinoma markers" including TTF1, CK7, Napsin A and PE10 in p-SQCC compared to c-SQCC

\begin{tabular}{lccc}
\hline IHC markers & p-SQCC & c-SQCC & P \\
\hline TTF1+ & $32 / 224(14.3 \%)$ & $30 / 386(7.8 \%)$ & 0.010 \\
CK7+ & $58 / 165(35.2 \%)$ & $52 / 256(20.3 \%)$ & 0.001 \\
Napsin A+ & $12 / 92(13.0 \%)$ & $9 / 126(7.1 \%)$ & 0.145 \\
PE10+ & $15 / 220(6.8 \%)$ & $18 / 381(4.7 \%)$ & 0.278 \\
\hline
\end{tabular}

IHC, immunohistochemical; $\mathrm{p}$-SQCC, peripheral lung squamous cell carcinoma; c-SQCC, central lung squamous cell carcinoma.

Table 4 Gene expression levels of "adenocarcinoma markers" and "squamous marker" in p-SQCC compared to c-SQCC

\begin{tabular}{cccc}
\hline Genes & $\begin{array}{c}\text { p-SQCC }(\mathrm{n}=60), \\
\text { high expression } \\
(\%)^{*}\end{array}$ & $\begin{array}{c}\text { c-SQCC }(\mathrm{n}=60), \\
\text { high expression } \\
(\%)\end{array}$ & $P$ \\
\hline Adenocarcinoma markers & $25(41.7 \%)$ & 0.068 \\
TTF1 & $35(58.3 \%)$ & $29(48.3 \%)$ & 0.715 \\
CK7 & $31(51.7 \%)$ & $22(36.7 \%)$ & 0.003 \\
SPA & $38(63.3 \%)$ & $30(50.0 \%)$ & 1.000 \\
CK8 & $30(50.0 \%)$ & & \\
Squamous marker & & $36(60.0 \%)$ & 0.028 \\
TP63 & $24(40.0 \%)$ & & \\
\hline
\end{tabular}

*, median expression level was used as the cutoff value for separating high and low expression. $\mathrm{p}-\mathrm{SQCC}$, peripheral lung squamous cell carcinoma; c-SQCC, central lung squamous cell carcinoma.

proportion of $\mathrm{p}-\mathrm{SQCC}$ displayed positive expression of TTF1 ( $\mathrm{P}=0.010)$ and CK7 $(\mathrm{P}=0.001)$. Samples from p-SQCC also showed higher frequency of positive Napsin $\mathrm{A}$ and PE10 staining, although statistical significance was not reached.

We also compared the gene expression levels of "adenocarcinoma markers" (including TTF1, CK7, SPA and CK8) and "squamous marker" (TP63) between p-SQCC and c-SQCC using qRT-PCR (Table 4). Significantly higher mRNA expression of SPA $(\mathrm{P}=0.003)$ was observed in $\mathrm{p}-\mathrm{SQCC}$, whereas c-SQCC showed higher mRNA expression of TP63 $(\mathrm{P}=0.028)$.

\section{Morphological characteristics of p-SQCC}

Among the 119 p-SQCC samples reviewed for morphological characteristics, 59 showed keratinization and 60 were nonkeratinizing. Basaloid subtype was found in 14 cases. According to tumor growth patterns, 2 were classified as alveolar space-filling type, 77 were expanding type, and 40 were combined type. Tumor cell budding and single-cell invasion was observed in 28 and 48 samples, respectively. The two cases classified as alveolar spacefilling type were both stage I with no lymph node metastasis or lymphovascular invasion, and had no recurrence. No significant associations were observed between morphological characteristics and OS (Figure S1).

\section{Discussion}

SQCC is typically known as a central lung lesion, while ADC is usually peripherally located. Our previous studies showed in vivo evidence of lung ADC to SQCC transdifferentiation $(6,7)$. This leads to our hypothesis that p-SQCC might have some "adenocarcinoma features" compared to c-SQCC. Some previous studies have indicated distinct clinicopathologic characteristics, morphology and immunophenotypes between p-SQCC and c-SQCC $(2,15,16)$. However, to our knowledge, this study is the first to comprehensively assess the clinicopathologic and morphologic features, prognosis, status of recurrent driver mutations along with immunohistochemical markers in $\mathrm{p}-\mathrm{SQCC}$.

In the clinicopathologic analysis, we found a positive correlation between p-SQCC and female gender as well as never-smoking status. These were both typical characteristics of lung ADC. Funai and colleagues (2) compared the clinicopathologic characteristics between 109 p-SQCCs and 95 c-SQCCs. They found that patients with p-SQCC were associated with older age, lower pathologic stages and incidence of lymph node metastasis. In this study, we also found patients with p-SQCC were significantly older than those with c-SQCC. Consistent with previous studies, we also observed p-SQCC patients were diagnosed at earlier stages than c-SQCC patients. In this study, we performed survival comparison between p-SQCC and c-SQCC, and revealed that the two subtypes had comparable RFS and OS in all patients as well as in stage I, stage II or stage III patients when analyzed separately. Funai and colleagues also found that survival proportions did not differ significantly between p-SQCC and c-SQCC although p-SQCC patients were associated with a lower pathologic stage and lower lymph node metastasis (2). Mizushima and colleagues also reported that p-SQCC and c-SQCC 
patients had no significant difference in the prognosis (17).

This study was the first to compare the status of a comprehensive panel of recurrent driver mutations between p-SQCC and c-SQCC. Interestingly, p-SQCC had a significantly higher proportion of known driver mutations than c-SQCC. Prevalence of EGFR mutations in p-SQCC was also significantly higher than that in c-SQCC. In addition, the frequency of KRAS mutations and HER2 mutations were not statistically significantly different, but there was a trend towards higher frequency in $\mathrm{p}-\mathrm{SQCC}$ compared to c-SQCC. The relatively higher proportion of EGFR, KRAS and HER2 mutations in p-SQCC also indicated "adenocarcinoma features". Furthermore, our results might have implications for clinical trials of targeted therapies for lung SQCC.

Collectively, we found that p-SQCC shared some features with $\mathrm{ADC}$ when compared to c-SQCC, including peripheral tumor location, female gender, never-smoking status and a higher proportion of EGFR mutations. Our IHC analysis further showed that positive staining of "adenocarcinoma markers" including TTF1, CK7, Napsin $\mathrm{A}$ and PE10 was found to be more prevalent in p-SQCC than c-SQCC. Finally, we compared the mRNA expression level of "adenocarcinoma markers" and "squamous marker" in p-SQCC and c-SQCC, and found that p-SQCC had significantly higher gene expression of SPA (ADC marker), while c-SQCC showed higher gene expression of TP63 (squamous marker).

In conclusion, lung p-SQCC had distinctive clinicopathologic characteristics and molecular features compared to c-SQCC, but showed some similarity with ADC.

\section{Acknowledgements}

Funding: This work was supported by the National Natural Science Foundation of China (81401886, 81422029, 81472173 and 81372525), the Key Project of Science and Technology Commission of Shanghai Municipality (JGGG1302, 14495810800), the Shen-Kang Center Project (SKMB1201), the Shanghai Hospital Development Center (SHDC12012308) and the Health and Family Planning Commission of Shanghai Municipality (2013ZYJB0301).

\section{Footnote}

Conflicts of Interest: The authors have no conflicts of interest to declare.
Ethical Statement: The study was approved by the Institutional Review Board of Fudan University Shanghai Cancer Center (No. 090977-1) and written informed consent was obtained from all patients.

\section{References}

1. Asamura H, Nakayama H, Kondo H, et al. Lymph node involvement, recurrence, and prognosis in resected small, peripheral, non-small-cell lung carcinomas: are these carcinomas candidates for video-assisted lobectomy? J Thorac Cardiovasc Surg 1996;111:1125-34.

2. Funai K, Yokose T, Ishii G, et al. Clinicopathologic characteristics of peripheral squamous cell carcinoma of the lung. Am J Surg Pathol 2003;27:978-84.

3. Sakurai H, Asamura H, Watanabe S, et al. Clinicopathologic features of peripheral squamous cell carcinoma of the lung. Ann Thorac Surg 2004;78:222-7.

4. Watanabe S, Oda M, Go T, et al. Should mediastinal nodal dissection be routinely undertaken in patients with peripheral small-sized ( $2 \mathrm{~cm}$ or less) lung cancer? Retrospective analysis of 225 patients. Eur J Cardiothorac Surg 2001;20:1007-11.

5. Zhang Y, Sun Y, Shen L, et al. Predictive factors of lymph node status in small peripheral non-small cell lung cancers: tumor histology is more reliable. Ann Surg Oncol 2013;20:1949-54.

6. Li F, Han X, Li F, et al. LKB1 Inactivation Elicits a Redox Imbalance to Modulate Non-small Cell Lung Cancer Plasticity and Therapeutic Response. Cancer Cell 2015;27:698-711.

7. Han X, Li F, Fang Z, et al. Transdifferentiation of lung adenocarcinoma in mice with Lkb1 deficiency to squamous cell carcinoma. Nat Commun 2014;5:3261.

8. Travis WD, Colby TV, Corrin B, et al. editors. Histological Typing of Lung and Pleural Tumours (International Histological Classification of Tumours). Geneva: World Health Organization, 1999:1-55.

9. Detterbeck FC, Boffa DJ, Tanoue LT. The new lung cancer staging system. Chest 2009;136:260-71.

10. Travis WD, Brambilla E, Nicholson AG, et al. The 2015 World Health Organization Classification of Lung Tumors: Impact of Genetic, Clinical and Radiologic Advances Since the 2004 Classification. J Thorac Oncol 2015;10:1243-60.

11. Weichert W, Kossakowski C, Harms A, et al. Proposal of a prognostically relevant grading scheme for pulmonary 
squamous cell carcinoma. Eur Respir J 2016;47:938-46.

12. Zhang Y, Li J, Wang R, et al. The prognostic and predictive value of solid subtype in invasive lung adenocarcinoma. Sci Rep 2014;4:7163.

13. Pan Y, Wang R, Ye T, et al. Comprehensive analysis of oncogenic mutations in lung squamous cell carcinoma with minor glandular component. Chest 2014;145:473-9.

14. Pan Y, Zhang Y, Li Y, et al. ALK, ROS1 and RET fusions in 1139 lung adenocarcinomas: a comprehensive study of common and fusion pattern-specific clinicopathologic, histologic and cytologic features. Lung Cancer 2014;84:121-6.

Cite this article as: Zhang Y, Zheng D, Li Y, Pan Y, Sun Y, Chen H. Comprehensive investigation of clinicopathologic features, oncogenic driver mutations and immunohistochemical markers in peripheral lung squamous cell carcinoma. J Thorac Dis 2017;9(11):4434-4440. doi: 10.21037/jtd.2017.10.43
15. Saijo T, Ishii G, Nagai K, et al. Differences in clinicopathological and biological features between central-type and peripheral-type squamous cell carcinoma of the lung. Lung Cancer 2006;52:37-45.

16. Hayashi T, Sano H, Egashira R, et al. Difference of morphology and immunophenotype between central and peripheral squamous cell carcinomas of the lung. Biomed Res Int 2013;2013:157838.

17. Mizushima Y, Yamashita R, Kusajima Y, et al. Prognostic comparison between peripheral and central types of squamous cell carcinoma of the lung in patients undergoing surgical resection. Oncol Rep 2000;7:319-22. 


\section{Supplementary}

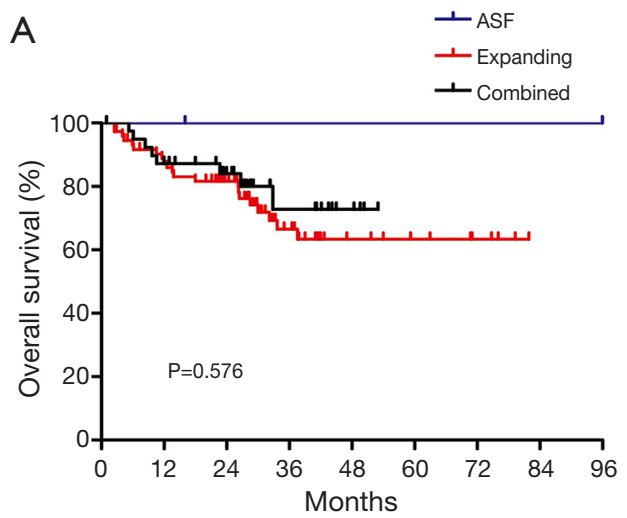

B

C
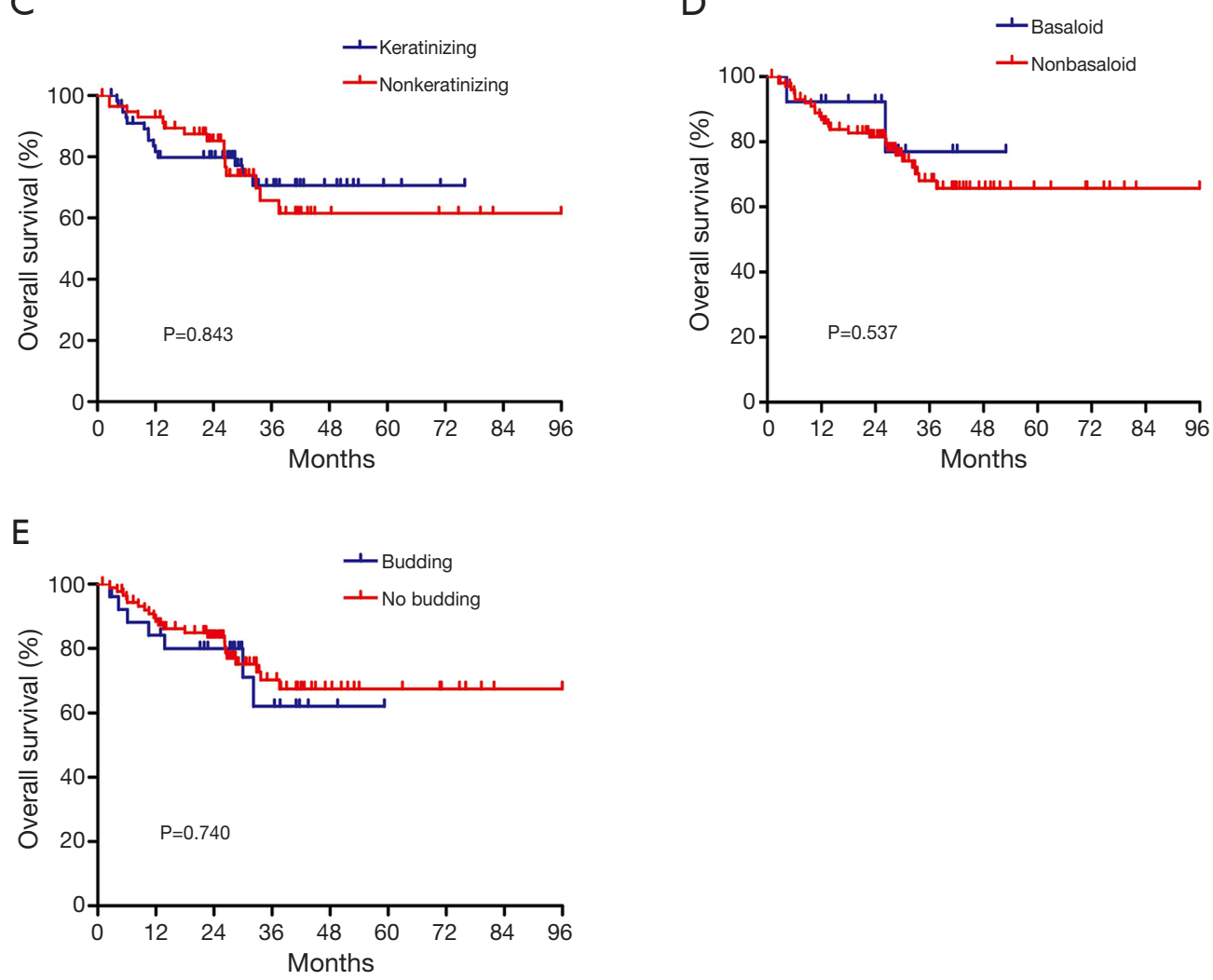

Figure S1 Associations of morphological characteristics with overall survival in 119 peripheral lung squamous cell carcinoma. ASF, alveolar space-filling. 